\title{
SHARP ESTIMATES IN SOME INEQUALITIES OF ZYGMUND TYPE FOR RIESZ TRANSFORMS
}

\author{
JORGE AARÃO AND MICHAEL D. O'NEILL \\ (Communicated by Edward C. Waymire)
}

\begin{abstract}
Sharp constant versions of two endpoint inequalities for Riesz transforms are derived using probabilistic methods.
\end{abstract}

\section{INTRODUCTION}

Sharp constants for the $L^{p}$ inequalities for singular integral operators $T$,

$$
\|T f\|_{p} \leq C_{p}\|f\|_{p}
$$

were found independently by Pichorides [7] and Cole [5] in the prototypical case, where $T$ is the conjugation operator $T u=u^{*}$ on harmonic functions in the unit disk. Cole's work on the conjugation operator in uniform algebras was unpublished but later appeared in the monograph [5]. In the case that $T$ is the Riesz transform, Bañuelos and Wang, in [1], found the sharp constants in (1) by probabilistic methods following upon ideas of Burkholder (see for example [3]), and Gundy and Varopolous [6.

The inequalities in (1) do not hold for $p=1$ or for $p=\infty$, but when $T$ is the conjugation operator, Zygmund proved that

$$
\int|T f| \leq C_{1}+C_{2} \int|f| \log ^{+}|f|
$$

as a substitute for the case $p=1$, and for $\|f\|_{\infty} \leq 1$ he showed that

$$
\int e^{c_{1}|T f|} \leq c_{2}
$$

See [9] for (2) and [10] volume I pg. 254 for (3). Calderon and Zygmund extended (2) and (3) to more general singular integral operators in [4] by real variable methods. Our purpose here is to give a version of (2) which is sharp for the constant $C_{2}$ and a version of (3) which is sharp for the constant $c_{1}$ in the case when $T$ is the Riesz transform. In the case of the conjugation operator, the sharp value of $C_{2}<\pi / 2$ was shown by Zygmund. The other sharp values of these constants for the conjugation operator were found independently by Cole and Pichorides. The method we will use is a mixture of the ideas from Cole's work and from the probabilistic methods of Banuelos and Wang. In section 2 we provide background on the probabilistic techniques which will be used. In section 3 we prove the sharp Riesz transform version of (3) and in section 4 we prove the sharp Riesz transform version of (2).

Received by the editors May 26, 2011.

2010 Mathematics Subject Classification. Primary 26D07, 42B20, 60H30.

(C)2012 American Mathematical Society Reverts to public domain 28 years from publication 


\section{Riesz transforms And STOChastic IntEgRALS}

Our main tools for proving the inequalities in the next two sections will be the Gundy-Varopoulos probabilistic interpretation of the Riesz transforms, see [6], and results of Bañuelos and Wang on differentially subordinate orthogonal martingales from [1. We provide a brief outline here and refer to the above papers for complete details.

Let $\Omega$ denote the set of paths in $\mathbb{R}_{+}^{(n+1)}=\left\{\left(x_{1}, \ldots, x_{n}, y\right): x_{i} \in \mathbb{R}, y \geq 0\right\}$ parametrized on $-\infty<t \leq 0$ which are at $y=0$ when $t=0$ and tend to the point at $\infty$ when $t \rightarrow-\infty$. For each $\omega \in \Omega$ let $W_{t}(\omega)=\omega(t)$ and for each $t$ let $\mathcal{F}_{t}$ denote the $\sigma$-algebra generated by the random variable $W_{t}$. Let $\mathcal{F}$ denote the smallest $\sigma$-algebra containing all the $\mathcal{F}_{t}$. Let

$$
\tau_{a}(\omega)=\inf \left\{s: y_{s}(\omega)=a\right\},
$$

where $y_{s}$ denotes the vertical component of $W_{s}$ and denote by $\mathcal{F}^{\tau}$ the $\sigma$-algebra generated by the random variables $\left\{W_{\tau+t}: t \geq 0\right\}$. Define a $\sigma$-finite measure $P^{\tau}$ on $\mathcal{F}^{\tau}$ by requiring that

$$
P^{\tau}\left(A_{G, t}\right)=\int_{\{y=a\}} \operatorname{Prob}\left\{B_{t}^{x} \in G\right\} d x
$$

for any set of the form

$$
A_{G, t}=\left\{\omega: W_{\tau+t}(\omega) \in G\right\},
$$

where $G$ is a Borel subset of $\mathbb{R}^{(n+1)}, t \geq 0$ and $B_{t}^{x}$ denotes the usual $(n+1)$ dimensional Brownian motion. By the strong Markov property of Brownian motion and the fact that $\mathcal{F}^{\tau} \subset \mathcal{F}^{\tau^{\prime}}$ for $\tau \leq \tau^{\prime}$, the measure $P^{\tau^{\prime}}$ is an extension of $P^{\tau}$ and we thus obtain a measure defined on the $\sigma$-algebra generated by the union of all the $\mathcal{F}^{\tau}$. Gundy and Varopolous note that this measure is defined on the $\sigma$-algebra generated by the random variables $W_{t}$ for $t \leq 0$ and that it is invariant under translations and rotations of the boundary of $\mathbb{R}^{(n+1)}$ and invariant up to a time change under dilations of $\mathbb{R}^{(n+1)}$. Heuristically, the process $W_{t}$ thus defined for $-\infty<t \leq 0$ is a Brownian motion started at $\{y=+\infty\}$ at time $t=-\infty$ with the Lebesgue measure as its initial distribution and which terminates at $t=0$ upon hitting the boundary of the upper half-space. All the rules of stochastic integration and the Itô calculus apply to this process. In particular, for any function $u$ harmonic in $\mathbb{R}^{(n+1)}$, vanishing at $\infty$ we have

$$
u\left(W_{t}\right)=\int_{-\infty}^{t} \nabla u\left(W_{s}\right) \cdot d W_{s} .
$$

Let $E$ denote expectation with respect to the measure constructed above. Gundy and Varopolous prove that if $f$ is a function in the Schwartz class in $\mathbb{R}^{n}$ and $u$ is its harmonic extension to $\mathbb{R}_{+}^{(n+1)}$, then for any $(n+1) \times(n+1)$ matrix $A$, the operator $\mathcal{A}$ defined by

$$
\mathcal{A}(f)\left(W_{0}\right)=E\left[\int_{-\infty}^{0} A \nabla u \cdot d W \mid W_{0}\right]
$$

has the form

$$
\mathcal{A}=\sum_{i=0}^{n} a_{i} R_{i}+\sum_{j, k=1}^{n} b_{j k} R_{j} R_{k}
$$


where $R_{j}$ denotes the $j$ th Riesz transform,

$$
R_{j} f(x)=c_{n} \text { p.v. } \int_{\mathbb{R}^{n}} \frac{x_{j}-y_{j}}{|x-y|^{n+1}} f(y) d y
$$

with

$$
c_{n}=\frac{\Gamma\left(\frac{(n+1)}{2}\right)}{\pi^{\frac{(n+1)}{2}}} .
$$

Bañuelos and Wang note that with the matrix $A^{j}$ defined by

$$
A_{r q}^{j}= \begin{cases}1 & r=1, q=j+1 \\ -1 & r=j+1, q=1 \\ 0 & \text { otherwise }\end{cases}
$$

we have $\left\|A^{j}\right\|=1, A^{j} v \cdot v=0$ for all $v$ and

$$
\mathcal{A}^{j}(f)(x)=R_{j} f(x) .
$$

It follows that the two martingales

$$
X_{t}=u\left(W_{t}\right)=\int_{-\infty}^{t} \nabla u\left(W_{s}\right) \cdot d W_{s}
$$

and

$$
Y_{t}=\int_{-\infty}^{t} A^{j} \nabla u\left(W_{s}\right) \cdot d W_{s}
$$

are orthogonal $\left(\langle X, Y\rangle_{t}=0\right)$ and, since

$$
\langle Y\rangle_{t}-\langle Y\rangle_{s} \leq\left\|A^{j}\right\|^{2}\langle X\rangle_{t}-\left\|A^{j}\right\|^{2}\langle X\rangle_{s},
$$

that $\langle X\rangle_{t}-\langle Y\rangle_{t}$ is a non-decreasing function of $t$. This is the Bañuelos-Wang differential subordination condition. Here $\rangle \cdot\langle$ denotes the usual quadratic variation of a martingale. See [1] and the references therein.

\section{A SHARP EXPONENTIAL TYPE INEQUALITY}

Theorem 1. Let $f$ be a $C^{\infty}$ function with support contained in the unit ball $B$ of $\mathbb{R}^{n}$ and suppose that $\|f\|_{\infty} \leq 1$. Then

$$
\int_{B} e^{\alpha\left|R_{j} f\right|} d x \leq \frac{C|B|}{\cos \alpha}
$$

for each $\alpha<\frac{\pi}{2}$ and some universal constant $C>0$ independent of $f$ and $\alpha$.

It follows easily that

$$
\left|\left\{\left|R_{j} f\right|>\lambda\right\} \cap B\right| \leq C_{\alpha} e^{-\alpha \lambda} .
$$

Proof of Theorem 1. Fix $0<\alpha<\frac{\pi}{2}$ and define

$$
g(x, y)=\cos (\alpha x) \cosh (\alpha y) .
$$

Following [5, pg. 122], for each $-1<x<1$ and $z=x+i y$ we have

$$
e^{ \pm \alpha y} \cos \alpha \leq e^{ \pm \alpha} \cos (\alpha x)=\operatorname{Re}\left(e^{\mp i \alpha z}\right)
$$

and therefore

$$
e^{\alpha|y|} \frac{\cos \alpha}{2} \leq g(x, y)
$$


Let $X_{t}=u\left(W_{t}\right)$ and $Y_{t}=\int_{-\infty}^{t} A^{j} \nabla u\left(W_{s}\right) \cdot d W_{s}$, where $W_{t}$ is the Gundy-Varopoulos background radiation and $A^{j}$ is the matrix corresponding to the $j$ th Riesz transform as discussed in the previous section. By Itô's formula we have

$$
\begin{aligned}
g\left(X_{t}, Y_{t}\right)=1 & +\int_{-\infty}^{t} g_{x}\left(X_{s}, Y_{s}\right) d X_{s}+\int_{-\infty}^{t} g_{y}\left(X_{s}, Y_{s}\right) d Y_{s} \\
& +\frac{1}{2} \int_{-\infty}^{t} g_{x x}\left(X_{s}, Y_{s}\right) d\langle X\rangle_{s}+\frac{1}{2} \int_{-\infty}^{t} g_{y y}\left(X_{s}, Y_{s}\right) d\langle Y\rangle_{s} \\
& +\int_{-\infty}^{t} g_{x y}\left(X_{s}, Y_{s}\right) d\langle X, Y\rangle_{s} .
\end{aligned}
$$

The last term vanishes by the orthogonality condition. The expected values of the second and third terms are zero by the martingale property of the stochastic integral. Because, for any real numbers $h, k$,

$$
g_{x x} h^{2}+g_{y y} k^{2}=-\left(\alpha^{2} \cos (\alpha x) \cosh (\alpha y)\right)\left(h^{2}-k^{2}\right),
$$

we have

$$
\begin{aligned}
& \int_{-\infty}^{t} g_{x x}\left(X_{s}, Y_{s}\right) d\langle X\rangle_{s}+\int_{-\infty}^{t} g_{y y}\left(X_{s}, Y_{s}\right) d\langle Y\rangle_{s} \\
= & -\int_{-\infty}^{t}\left(\alpha^{2} \cos \left(\alpha X_{s}\right) \cosh \left(\alpha Y_{s}\right)\right) d\left(\langle X\rangle_{s}-\langle Y\rangle_{s}\right) .
\end{aligned}
$$

This is non-positive by the differential subordination condition. It follows that $E\left(g\left(X_{t}, Y_{t}\right)-1\right) \leq 0$ and therefore that

$$
\int_{\mathbb{R}^{n}}\left(g\left(f, R_{j} f\right)-1\right) d x \leq 0
$$

since the initial distribution of $W$ is the Lebesgue measure.

Because $f$ is supported in $B$ and $R_{j} f=O\left(\frac{1}{|x|^{n}}\right)$ for large $x$, the above integrand is $O\left(\frac{1}{|x|^{2 n}}\right)$ and thus by (4),

$$
\int_{B} e^{\alpha\left|R_{j} f\right|} d x \leq \frac{C|B|}{\cos \alpha}
$$

\section{A Sharp $L$ LOG $L$ inequality}

Theorem 2. Suppose $f \geq 0$ and supported in a finite ball $B$ in $\mathbb{R}^{n}$. Then for any $\gamma>\frac{2}{\pi}$ there is a $\beta \geq 0$ such that

$$
\int_{B}\left|R_{j} f(x)\right| d m \leq \beta+\gamma \int_{B} f \log ^{+} f d m,
$$

where $R_{j}$ is the jth Riesz transform.

Proof. Let $\epsilon>0$ be fixed and let $\alpha>0$ and $\delta>0$ be parameters to be adjusted in what follows. Other constants appearing in the proof are numerical and may change from line to line in ways which do not affect the final form of the theorem. Subscripts or functional arguments to constants will be used as needed to indicate their origin in a previous step.

Define a function $g(x, y) \in C^{\infty}\left(\mathbb{R}^{2}\right)$ satisfying the following conditions: 
(a) $g(x, y)=\frac{\delta x}{2} \log \left(x^{2}+y^{2}\right)-\delta y \arctan \left(\frac{y}{x}\right)-\alpha x$

for values of $(x, y)$ such that $x>0$ and $x^{2}+y^{2} \geq 4 \epsilon^{2}$.

(b) $g$ vanishes if $x<-\epsilon$ or if $x^{2}+y^{2}<\epsilon^{2}$.

(c) $\left|g_{x}\right|$ and $\left|g_{y}\right|$ are bounded by $C\left(\delta \log \frac{1}{\epsilon}+\alpha\right)$ for $\epsilon^{2} \leq x^{2}+y^{2} \leq 4 \epsilon^{2}$.

(d) $\left|g_{x x}\right|,\left|g_{x y}\right|$ and $\left|g_{y y}\right|$ are bounded by $\frac{C}{\epsilon}\left(\delta \log \frac{1}{\epsilon}+\alpha\right)$ for $\epsilon^{2} \leq x^{2}+y^{2} \leq 4 \epsilon^{2}$. Given $\delta>\frac{2}{\pi}$, choose $\alpha>0$ so that

$$
g(x, y) \leq \delta x \log x-|y|
$$

for all $(x, y)$ such that $x>0$ and $x^{2}+y^{2} \geq 4 \epsilon^{2}$.

Let $u$ be the harmonic extension of $f$ given by the Poisson integral. As before, $X_{t}=u\left(W_{t}\right)$ and $Y_{t}=\int_{-\infty}^{t} A^{j} \nabla u\left(W_{s}\right) \cdot d W_{s}$.

By Itô's formula we have

$$
\begin{aligned}
g\left(X_{t}, Y_{t}\right)=g\left(X_{-\infty}, Y_{-\infty}\right) & +\int_{-\infty}^{t} g_{x}\left(X_{s}, Y_{s}\right) d X_{s}+\int_{-\infty}^{t} g_{y}\left(X_{s}, Y_{s}\right) d Y_{s} \\
& +\frac{1}{2} \int_{-\infty}^{t} g_{x x}\left(X_{s}, Y_{s}\right) d\langle X\rangle_{s}+\frac{1}{2} \int_{-\infty}^{t} g_{y y}\left(X_{s}, Y_{s}\right) d\langle Y\rangle_{s} \\
& +\int_{-\infty}^{t} g_{x y}\left(X_{s}, Y_{s}\right) d\langle X, Y\rangle_{s} .
\end{aligned}
$$

The first term is zero and the last term vanishes by the orthogonality condition. To prove our inequality, we will need to take the expectation of $g\left(X_{t}, Y_{t}\right)$ at $t=0$, but we again note that the second and third terms above are martingales with expectation zero. We are thus led to consider the expected value of the fourth and fifth terms on the right-hand side above.

For any real numbers $h, k$, we have, for $x^{2}+y^{2} \geq 4 \epsilon^{2}$,

$$
g_{x x} h^{2}+g_{y y} k^{2}=\frac{\delta x}{x^{2}+y^{2}}\left(h^{2}-k^{2}\right) .
$$

Letting $A_{\epsilon}=\left\{t: X_{t}^{2}+Y_{t}^{2} \geq \epsilon^{2}\right\}$, it follows that

$$
\int_{-\infty}^{t} g_{x x}\left(X_{s}, Y_{s}\right) d\langle X\rangle_{s}+\int_{-\infty}^{t} g_{y y}\left(X_{s}, Y_{s}\right) d\langle Y\rangle_{s}=\mathrm{I}+\mathrm{II},
$$

where

$$
\mathrm{I}=\int_{A_{\epsilon}} \frac{\delta x}{x^{2}+y^{2}} d\left(\langle X\rangle_{s}-\langle Y\rangle_{s}\right)
$$

and

$$
\mathrm{II}=\int_{A_{\epsilon}^{c}} g_{x x}\left(X_{s}, Y_{s}\right) d\langle X\rangle_{s}+g_{y y}\left(X_{s}, Y_{s}\right) d\langle Y\rangle_{s} .
$$

The term I is non-negative by the differential subordination condition, and for the term II we have

$$
E(\mathrm{II}) \geq-C \frac{\delta}{\epsilon}\left(\log \frac{1}{\epsilon}+\alpha\right) E\left(\int_{A_{\epsilon}^{c}} d\left(\langle X\rangle_{s}+\langle Y\rangle_{s}\right)\right) .
$$

The martingales $X_{\langle X\rangle_{s}}$ and $Y_{\langle Y\rangle_{s}}$ are Brownian motions. So by the time-scaling property of Brownian motion we have

$$
E(\mathrm{II}) \geq-C a_{0} \delta \epsilon\left(\log \frac{1}{\epsilon}+\alpha\right)
$$


where $a_{0}$ denotes the expected escape time for Brownian motion from a Euclidean half-space started one unit distance from the boundary.

It follows from (5) that

$$
\begin{aligned}
-C \delta \epsilon\left(\log \frac{1}{\epsilon}+\alpha\right) & \leq\left. E\left(g\left(X_{t}, Y_{t}\right)\right)\right|_{t=0} \\
& =\int_{\mathbb{R}^{n}} g\left(f, R_{j} f\right) d x \\
& \leq \mathrm{III}+\mathrm{IV},
\end{aligned}
$$

where

$$
\mathrm{III}=\int_{\epsilon \leq\left\|\left(f, R_{j} f\right)\right\| \leq 2 \epsilon} g\left(f, R_{j} f\right) d x
$$

and

Consequently, we have

$$
\mathrm{IV}=\int_{\left\|\left(f, R_{j} f\right)\right\|>2 \epsilon}\left(\delta f \log f-\left|R_{j} f\right|\right) d x .
$$

$$
\begin{aligned}
\int_{B}\left|R_{j} f\right| d x & \leq \int_{\left\|\left(f, R_{j} f\right)\right\|>2 \epsilon}\left|R_{j} f\right| d x+2 \epsilon|B| \\
& \leq c(\epsilon, \delta, \alpha)+\mathrm{III}+2 \epsilon|B|+\int_{B} \delta f \log ^{+} f d x .
\end{aligned}
$$

To estimate III, note that $\left\{\epsilon<\left\|\left(f, R_{j} f\right)\right\|<2 \epsilon\right\} \subset B \cup\left\{\epsilon<\left|R_{j} f\right|<2 \epsilon\right\}$ and that for large $|x|$ we have

$$
\left|R_{j} f(x)\right| \leq \frac{C}{|x|^{n}} \int_{B}|f| d y .
$$

For any $\eta>0$ there is $C_{\eta}>0$ such that for all $f \in L \log ^{+} L(B)$,

$$
\int_{B}|f| d y \leq C_{\eta}+\eta \int_{B} f \log ^{+} f d y
$$

It follows that

$$
\left|\left\{x: \epsilon<\left|R_{j} f(x)\right|\right\}\right| \leq \frac{C}{\epsilon}\left(C_{\eta}+\eta \int_{B} f \log ^{+} f d y\right) .
$$

Since $\left|g\left(f, R_{j} f\right)\right| \leq C \epsilon\left(\delta \log \frac{1}{\epsilon}+\alpha\right)$ on the set $\left\{\epsilon<\left\|\left(f, R_{j} f\right)\right\|<2 \epsilon\right\}$ we have

$$
\mathrm{III} \leq c(\eta, \epsilon, \alpha, \delta)+C\left(\delta \log \frac{1}{\epsilon}+\alpha\right) \eta \int_{B} f \log ^{+} f d y,
$$

and choosing $\eta$ small enough so that

$$
C\left(\delta \log \frac{1}{\epsilon}+\alpha\right) \eta+\delta<\gamma
$$

gives the conclusion of the theorem.

\section{ACKNOWLEDGMENT}

In his book Probabilistic Techniques in Analysis 2, R. Bass extends the background radiation approach to regular Brownian motion. The resulting method can no longer be applied to iterated Riesz transforms, but it should be applicable to the inequalities treated here. We thank the anonymous referee for this remark and for other helpful comments. 


\section{REFERENCES}

[1] R. Bañuelos and G. Wang, Sharp inequalities for martingales with applications to the Beurling-Ahlfors and Riesz transforms. Duke Math. J., 80 (3):575-600, 1995. MR.1370109 (96k:60108)

[2] R. F. Bass, Probabilistic Techniques in Analysis, Probability and its Applications (New York), Springer-Verlag, New York, 1995. MR1329542 (96e:60001)

[3] D. Burkholder, A sharp inequality for martingale transforms. Ann. Probab., 7:858-863, 1979. MR.542135 (80j:60067)

[4] A. Calderon and A. Zygmund, On the existence of certain singular integrals. Acta Math., 88:85-139, 1952. MR0052553(14:637f)

[5] T. Gamelin, Uniform Algebras and Jensen Measures. London Math. Society Lecture Notes Series, 32, Cambridge University Press, London and New York, 1978. MR521440 (81a:46058)

[6] R. Gundy and N. Varopoulos, Les transformations de Riesz et les intégrales stochastiques. (French), C.R. Acad. Sci. Paris Sér. A-B, 289 (1):A13-A16, 1979. MR545671 (82e:60089)

[7] S. Pichorides, On the best values of the constants in the theorems of M. Riesz, Zygmund and Kolmogorov. Studia Math., 44:165-179, 1972. MR0312140(47:702)

[8] E. Stein, Singular Integrals and Differentiability Properties of Functions. Princeton Mathematical Series, No. 30, Princeton University Press, New Jersey, 1970. MR0290095 (44:7280)

[9] A. Zygmund, Sur les fonctions conjugées. Fund. Math., 13:284-303, 1929.

[10] A. Zygmund, Trigonometric Series. Paperback edition, volumes I and II combined. Cambridge University Press, Cambridge, 1988. MR.933759 (89c:42001)

School of Mathematics and Statistics, University of South Australia, Mawson Lakes Boulevard, Mawson Lakes, 5070 SA, Australia

E-mail address: Jorge.Aarao@unisa.edu.au

Department of Mathematics, Claremont McKenna College, Claremont, California 91711

E-mail address: moneill@cmc.edu 